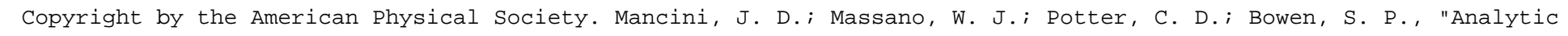

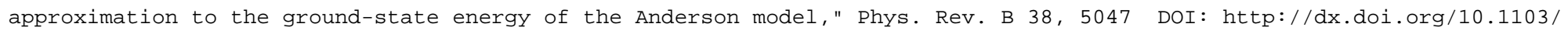
PhysRevB.38.5047

\title{
Analytic approximation to the ground-state energy of the Anderson model
}

\author{
Jay D. Mancini \\ Department of Physics, Fordham University, Bronx, New York 10458 \\ William J. Massano \\ Department of Science, State University of New York Maritime College, Fort Schuyler, \\ Bronx, New York 10465 \\ Charles D. Potter and Samuel P. Bowen \\ Physics Department, Virginia Tech, Blacksburg, Virginia 24061
}

(Received 21 March 1988)

\begin{abstract}
Using the nonperturbative Lanczos recursion scheme an analytic approximation to the groundstate energy of the single-impurity Anderson model is obtained. Calculations are carried out to a $5 \times 5$ matrix truncation. Comparisons are made with the exact Bethe-ansatz result.
\end{abstract}

\section{INTRODUCTION}

In recent years, lanthanides have received a great deal of attention as model systems for the phenomenon of mixed valence. ${ }^{1,2}$ These systems are characterized by localized $f$ orbitals of energy close to the Fermi energy which interact strongly with the conduction band. This occurs when the electronic configurations $[4 f]^{n}$ and $[4 f]^{n+1}$ are nearly degenerate in energy but are energetically isolated due to the large on-site Coulomb repulsion.

The simplest model Hamiltonian to describe such mixed-valence systems is the Anderson model. ${ }^{3}$ This model may describe both the mixed-valence system for a single rare-earth ion or a periodic lattice of ions. In this work we shall look only at the single-impurity Anderson model (SIAM) for which there are a number of exact results. Using the Bethe-ansatz technique, Wiegmann ${ }^{4}$ demonstrated that the Anderson Hamiltonian is exactly solvable under certain conditions. Kawakami and Okiji ${ }^{5}$ extended Wiegmann's work and obtained an explicit expression for the ground-state energy. Their calculations were performed by numerically solving a set of 200-300 coupled integral equations.

Recently, Mancini, Potter, and Bowen ${ }^{6}$ and Bowen and Mancini ${ }^{7}$ applied a finite-matrix numerical truncation scheme to study the ground state properties of the SIAM. For small truncations, $18 \times 18$, the results of this variational calculation ${ }^{8,9}$ were in close agreement with the exact Bethe-ansatz results. ${ }^{5}$ The nature of the calculation presented in this work is also variational. This approach utilizes an analytic truncation scheme rather than a numerical one. We shall employ the nonperturbative Lanczos scheme (tridiagonalization) ${ }^{10-12}$ to evaluate the ground-state energy of the SIAM.

A critical first step in this method is the choice of an approximate ground-state wave function $\left|\phi_{0}\right\rangle$ whose overlap with the exact ground state $\left|\psi_{0}\right\rangle$ is nonvanishing. As in any variational calculation, the choice of $\left|\phi_{0}\right\rangle$ is a vital determining factor in how rapidly the method converges to the exact ground state. However, maximizing the overlap of the approximate ground-state wave function with the exact wave function must be tempered with maintain- ing computational tractability.

The calculations up to the $4 \times 4$ truncation were done manually. An order of magnitude of the number of terms which needed to be evaluated is easily obtained. It is found that the diagonal matrix elements vary with powers of the Hamiltonian as $H, H^{3}, H^{5}, H^{7}$, and so forth. The Hamiltonian given by Eq. (2.1) contains five terms. Thus evaluation of the $4 \times 4$ truncation required the analysis of approximately $5^{7}=15625$ matrix elements. It is clear that this method becomes prohibitive very rapidly. $\mathrm{Re}$ cently Massano ${ }^{13}$ has developed a computer program which evaluates the necessary matrix elements symbolically. This program has served as both a check for the manual calculations as well as to supply the necessary matrix elements for the $5 \times 5$ truncation.

The paper is organized as follows. In Sec. II we define the Anderson Hamiltonian and outline the Lanczos scheme. This section will also briefly discuss the analytic structure of the tridiagonal energy matrix whose lowest eigenvalues yield an upper bound on the ground-state energy. In Sec. III we compare our result for the ground-state energy to that of Kawakami and Okiji. ${ }^{5}$ In Sec. IV we summarize with concluding remarks.

\section{METHOD}

The Anderson Hamiltonian ${ }^{3}$ was first proposed in 1961 to describe magnetic impurities in metals. A recent review of this model is given by Czycholl. ${ }^{14}$ The Anderson Hamiltonian is given by

$$
\begin{aligned}
H & =\sum_{k, s} n_{k, s} \varepsilon_{k, s}+\sum_{s} E_{s} N_{s}+U N_{\uparrow} N_{\downarrow} \\
& +\sum_{k, s} V(k)\left(c_{k, s}^{\dagger} f_{s}+f_{s}^{\dagger} c_{k, s}\right) .
\end{aligned}
$$

Here $c_{k s}^{\dagger}$ and $f_{s}^{\dagger}$ create conduction electrons in Bloch states $k, s$ and localized $f$ electrons, respectively. The occupation number operator for $d$ electrons is given by $n_{k s}=c_{k s}^{\dagger} c_{k s}$ and for the localized $f$ orbitals of $N_{s}=f_{s}^{\dagger} f_{s}$ where $s=\uparrow, \downarrow$ represents the spin states. The first term of the Hamiltonian describes the band electrons with energy 
$\varepsilon_{k s}$ of the host metal. The second term represents the energy of an electron in an (unperturbed) $f$ orbital. The parameter $U$ represents the Coulomb interaction energy between two $f$ electrons at the impurity site and $V(k)$ is the $f-d$ hybridization integral between the two bands taken in this work to be independent of $k$. We note similarities between the mixing term appearing in Eq. (2.1) and that which arises in band-structure calculations. In the latter case the transition between $d$ orbitals and the conduction band is treated by introducing the mixing of matrix elements. However, in the present case, the $f$ orbitals are localized for a finite time before hybridization occurs.

We choose our method of calculation to be the nonperturbative Lanczos recursion scheme (tridiagonalization). ${ }^{11,12}$ Let $\left|\phi_{i}\right\rangle$ be a normalized trial ket which has nonzero overlap with the exact ground-state wave function of the system under consideration. The Lanczos scheme is then generated by operation of the Hamiltonian on this ket yielding the following (tridiagonal) form:

$H\left|\phi_{i}\right\rangle=m_{i, i-1}\left|\phi_{i-1}\right\rangle+m_{i, i}\left|\phi_{i}\right\rangle+m_{i, i+1}\left|\phi_{i+1}\right\rangle$.

Physically, the new kets which are being generated represent higher-order particle-hole excitations from the ground state. Explicitly, for $\left|\phi_{i}\right\rangle=\left|\phi_{0}\right\rangle$ our trial groundstate wave function,

$$
H\left|\phi_{0}\right\rangle=m_{00}\left|\phi_{0}\right\rangle+m_{01}\left|\phi_{1}\right\rangle
$$

with

$$
\begin{aligned}
& m_{00}=\left\langle\phi_{0}|H| \phi_{0}\right\rangle, \\
& m_{01}=\left\langle\phi_{0}|H| \phi_{1}\right\rangle=m_{10},
\end{aligned}
$$

and from Eq. (2.3)

$$
\left|\phi_{1}\right\rangle=\frac{1}{m_{01}}\left(H-m_{00}\right)\left|\phi_{0}\right\rangle \text {. }
$$

Putting this result for $\left|\phi_{1}\right\rangle$ back into Eq. (2.4b) yields for $m_{01}$

$$
\begin{aligned}
m \sigma_{1}^{2} & =\left\langle\phi_{0}\left|H^{2}\right| \phi_{0}\right\rangle-m_{\sigma_{0}}^{2} \\
& =\left\langle\phi_{0}\left|H^{2}\right| \phi_{0}\right\rangle-\left\langle\phi_{0}|H| \phi_{0}\right\rangle^{2} .
\end{aligned}
$$

We see then that the matrix elements $m_{i j}$ of the tridiagonal energy matrix $\tilde{M}$ may be represented by ground-state matrix elements of powers of the Hamiltonian. The set of kets $\left|\phi_{i}\right\rangle$ are generated in a manner analogous to Eq. (2.5)

\section{RESULTS}

We have chosen the singlet initial state to be the empty impurity orbital and the filled Fermi sphere with pairs of scattering states with opposite spins

$$
\left|\phi_{0}\right\rangle=|F\rangle|0\rangle_{f} \text {. }
$$

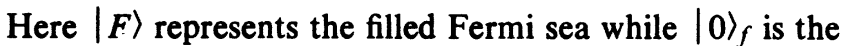
unoccupied localized $f$ orbital. We assume a unit density of conduction electrons

$$
\rho=\left\{\begin{array}{l}
1,-D / 2 \leq \varepsilon \leq D / 2, \\
0, \text { otherwise, }
\end{array}\right.
$$

where $D=1$ is the width of the conduction band. All calculations were performed for the particle-hole symmetric case where $U=-2 E_{f}$.

TABLE I. Using the recursion relation given by Eq. (2.2) the first five states are generated. Each successive state may be related to the initial state $\left|\phi_{0}\right\rangle=|F\rangle \mid 0_{f}$. Note that $m_{i, j}=\left\langle\phi_{i}|H| \phi_{j}\right\rangle$ and $m_{i, j}-m_{j, i}$

$$
\begin{aligned}
\left|\phi_{0}\right\rangle & =|F\rangle|0\rangle_{f} \\
\left|\phi_{1}\right\rangle & =\frac{1}{m_{10}}\left(H-m_{00}\right)\left|\phi_{0}\right\rangle \\
\left|\phi_{2}\right\rangle & =\frac{1}{m_{12}}\left[\left(H-m_{11}\right)\left|\phi_{1}\right\rangle-m_{10}\left|\phi_{0}\right\rangle\right] \\
& =\frac{1}{m_{12}}\left[\left(H-m_{11}\right) \frac{1}{m_{10}}\left(H-m_{00}\right)-m_{00}\right]\left|\phi_{0}\right\rangle \\
\left|\phi_{3}\right\rangle & =\frac{1}{m_{23}}\left[\left(H-m_{22}\right)\left|\phi_{2}\right\rangle-m_{21}\left|\phi_{1}\right\rangle\right] \\
& =\frac{1}{m_{23}}\left\{\left(H-m_{22}\right) \frac{1}{m_{12}}\left[\left(H-m_{11}\right) \frac{1}{m_{10}}\left(H-m_{00}\right)-m_{00}\right]-\frac{m_{21}}{m_{10}}\left(H-m_{00}\right)\right)\left|\phi_{0}\right\rangle \\
\left|\phi_{4}\right\rangle & \left.=\frac{1}{m_{34}}\left[\left(H-m_{33}\right)\left|\phi_{3}\right\rangle-m_{32}\left|\phi_{2}\right\rangle\right]\right] \\
& =\frac{1}{m_{34}}\left\{\left(H-m_{33}\right) \frac{1}{m_{23}}\left[\left(H-m_{22}\right) \frac{1}{m_{12}}\left(\left(H-m_{11}\right) \frac{1}{m_{10}}\left(H-m_{00}\right)-m_{00}\right) \frac{-m_{21}}{m_{10}}\left(H-m_{00}\right)\right]\right. \\
\left.\quad-\frac{m_{32}}{m_{12}}\left(\left(H-m_{11}\right) \frac{1}{m_{10}}\left(H-m_{00}\right)-m_{00}\right)\right\}\left|\phi_{0}\right\rangle & \\
\left|\phi_{n}\right\rangle & =\frac{1}{m_{n}-1, n}\left[\left(H-m_{n-1, n-1}\right)\left|\phi_{n-1}\right\rangle-m_{n-1, n-2}\left|\phi_{n-2}\right\rangle\right]
\end{aligned}
$$




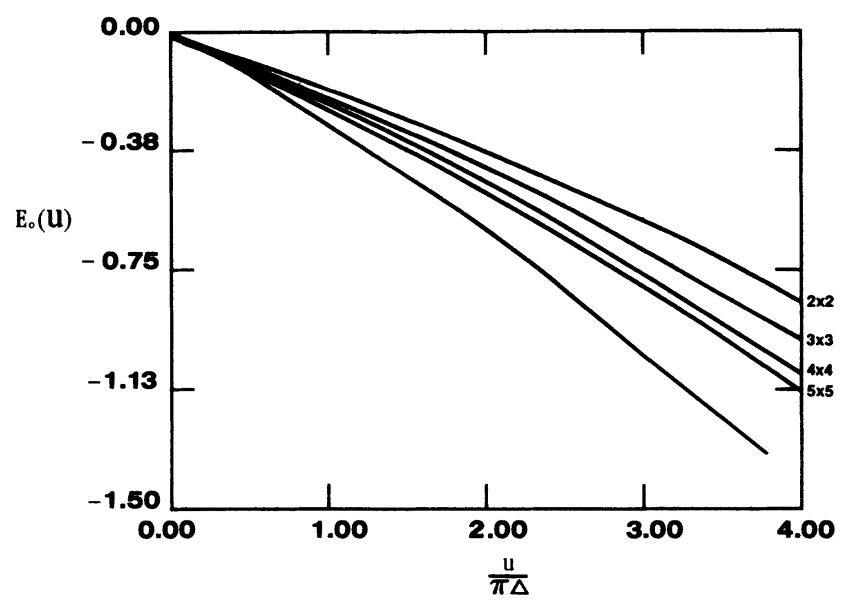

FIG. 1. Comparison of the ground-state energy $E_{0}(U)$ for various truncations (a) $2 \times 2$, (b) $3 \times 3$, (c) $4 \times 4$, and (d) $5 \times 5$. Also plotted is the exact Bethe-ansatz result.

Using the recursion relation (2.2) the energy matrix $\tilde{M}$ is generated. The eigenvalues of this matrix are then determined, the lowest of which represents an upper bound to the ground-state energy. Table I lists the states generated using (2.2). Actual calculations are carried out by relating each of the $\left|\phi_{n}\right\rangle$ back to $\left|\phi_{0}\right\rangle$ and then performing the necessary integrals over the flat density of states given in (3.2). However, a necessary intermediate step involves normal ordering the long strings of operators which arise from taking the powers of the Hamiltonian (2.1). A computer program developed by Massano ${ }^{13}$ is able to perform this normal ordering as well as carry out the necessary integrals. Details of this program will be given elsewhere. The matrix elements of the $4 \times 4$ truncation were first performed manually and then checked by computer. The $5 \times 5$ truncation was necessarily done by the computer.

The ground-state energy $E_{0}$, as a function of $U$, is plotted in Fig. 1 in units which are consistent with Ref. 5. Also plotted is the exact Bethe-ansatz result of Ref. 5 .

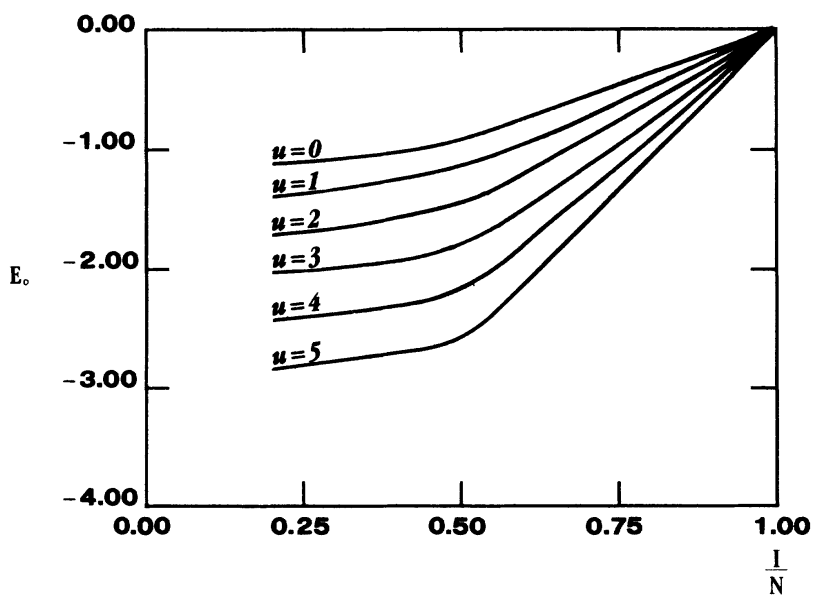

FIG. 2. Plot of the ground-state energy as a function of $1 / N$ where $N$ represents the size of the matrix truncation. Each curve represents a fixed value of $U$.

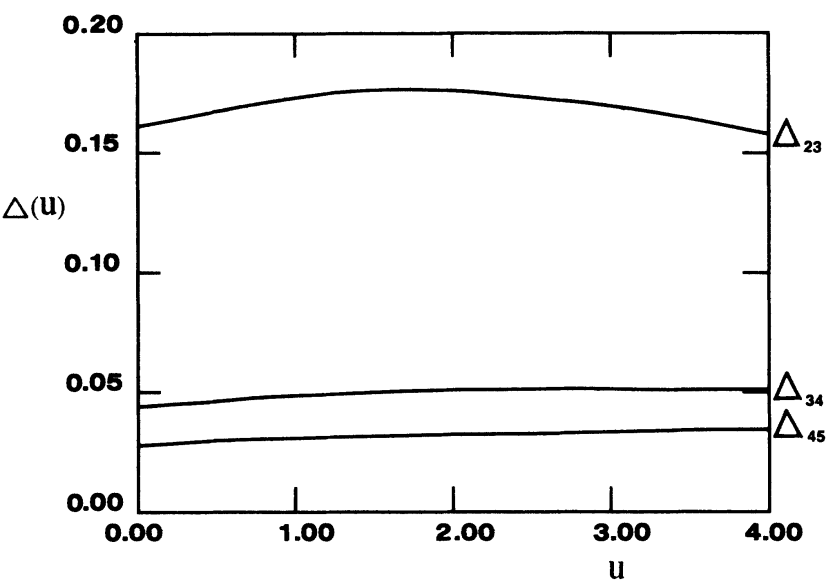

FIG. 3. Plot of the quantity $\Delta_{r p}=\left|E_{0}^{(r \times r)}(U)-E_{0}^{(p \times p)}(U)\right|$ which represents a qualitative measure of the rate of convergence for different truncations.

We see that the two results are in good agreement. A more rapid convergence to the exact result would be obtained by increasing the size of the (truncated) basis. This would entail generating a larger matrix $\tilde{M}$ according to (2.2) and determining its eigenvalues. The $5 \times 5$ truncation calculated here was performed on an IBM XT-type PC. The storage capacity of such a small machine was taken to its limit by the enormous amount of requisite operator algebra. Any further work would require the speed and memory capabilities of a large mainframe computer.

In Fig. 2 the ground-state energy $E_{0}^{(N \times N)}$ is plotted as a function of $1 / N$ where $N$ is the dimension of the matrix truncation. Each curve represents different values of the Coulomb repulsion $U$. We note the rapid convergence as witnessed by the nearly flat tail of each curve. It is evident that those points which would appear on the line where the abscissa vanishes represent our best estimate to the exact ground-state energy.

In Fig. 3 we have plotted the quantity

$$
\Delta_{r p}(U)=\left|E_{0}^{(r \times r)}(U)-E_{0}^{(p \times p)}(U)\right|,
$$

where $r$ and $p$ represent different truncation sizes. This quantity gives a qualitative measure of the rate of convergence by measuring the energy differences between successive truncations as a function of the parameter $U$. We see that the curves $\Delta_{34}$ and $\Delta_{45}$ are nearly flat and have values less than 0.5 . Thus truncating our basis at the $5 \times 5$ level yields convergence to within three decimal places. Hence we conclude that the large amount of labor necessary to proceed to higher truncations will not enhance the rate of convergence significantly. This reinforces our observation above that our best estimates for $E_{0}$ would be that obtained by extrapolation of those curves in Fig. 1.

\section{CONCLUSION}

Using the nonperturbative Lanczos scheme we have obtained an analytic result for the ground state of the 
single-impurity Anderson model. The choice of a trial ket $\left|\phi_{0}\right\rangle$ is dictated primarily by our desire to use a calculationally manageable starting point as well as satisfying the requirement that the ground state be singlet. The amount of requisite algebra dictated by the method rapidly becomes prohibitive for manual calculations and must be performed by computer. The method, which is variational in nature, is not restricted to any particular range of parameter space. We find this to be an advantage over perturbative schemes; however, the enormous number of operator manipulations required with this method warrants careful consideration.
${ }^{1}$ Valence Fluctuations in Solids, edited by L. M. Falicov, W. Henke, and M. B. Maple (North-Holland, Amsterdam, 1981).

${ }^{2}$ Valence Instabilities, edited by P. Wachter and H. Boppart (North-Holland, Amsterdam 1982).

${ }^{3}$ P. W. Anderson, Phys. Rev. 124, 41 (1961).

${ }^{4}$ P. B. Wiegmann, Phys. Lett. 80A, 16 (1980).

${ }^{5}$ N. Kawakami and A. Okiji, Phys. Lett. 86A, 483 (1981); J. Phys. Soc. Jpn. 5, 3192 (1982); 51, 2043 (1982).

${ }^{6}$ J. D. Mancini, C. D. Potter, and S. P. Bowen, J. Appl. Phys. 61, 3168 (1987).

${ }^{7}$ S. P. Bowen and J. D. Mancini (unpublished).
${ }^{8}$ C. M. Varma and Y. Yafet, Phys. Rev. B 13, 2950 (1976).

${ }^{9}$ O. Gunnarsson and K. Schonhammer, Phys. Rev. B 31, 4815 (1985).

${ }^{10}$ R. Haydock, V. Heine and M. J. Kelly, J. Phys. C 5, 2854 (1972).

11 J. D. Mancini and D. C. Mattis, Phys. Rev. B 28, 6061 (1983); 29, 6988 (1984); 31, 7440 (1985).

12J. D. Mancini and C. D. Potter, Nuovo Cimento 9, 481 (1987).

${ }^{13}$ W. J. Massano (unpublished).

${ }^{14}$ G. Czycholl, Phys. Rep. 143, 227 (1986), and references therein. 